\title{
FREE CONVECTION FLOW PAST A VERTICAL FLAT PLATE EMBEDDED IN A SATURATED POROUS MEDIUM
}

\author{
T. Y. NA \\ Department of Mechanical Engineering, University of Michigan-Dearborn, Dearborn. MI 48128. U.S.A. \\ and \\ 1. POP \\ Faculty of Mathematics, University of Cluj. R-3400 Cluj, CP 253 Romania \\ (Communicated by E. SOOS)

\begin{abstract}
A numerical solution for the free convection flow past a vertical semi-infinite flat plate embedded in a highly saturated porous medium by allowing the plate to have a non-uniform temperature or a non-uniform heat flux distributions has been developed. Both local heat transfer rate and excess surface temperature as a function of the distance along the plate are tabulated for a few cases of prescribed wall temperature and heat flux distributions. Such tabulations serve as a reference against which other approximate solutions can be compared in the future.
\end{abstract}

\section{INTRODUCTION}

THERE IS, at present, a good deal of information about laminar free convection boundary layers over isothermal surfaces for which the flow is similar. Approximate similarity transformations, approximate series and integral procedures and numerical finite-difference methods have all been employed in obtaining solutions of the laminar, steady state, free convection heat transfer from bodies with relatively simple geometry, e.g. flat plate, circular cylinder and sphere. However, problems of non-isothermal surfaces have received relatively little attention.

The problem of prediction of heat transfer is of importance in a number of geophysical and engineering applications, notably in studies of free convection flow from a heated vertical surface embedded in a saturated porous medium. The practice shows that porous media are very widely used for enclosing a heated body to keep its temperature; for they are considered to be useful in diminishing the natural convection which will otherwise occur intensely around the heated body. To make the heat insulation of the body more effective, it may be necessary to study the residual flow through porous medium induced by buoyancy and to estimate its effect on the heat transfer.

In a recent paper, Cheng and Minkowycz[1] have obtained similarity solutions for the free convection flow in a porous medium adjacent to a vertical plate with wall temperature being a power function of distance from the leading edge. A good description of the underlying physical assumptions as well as many interesting practical applications of this problem are also sketched in a series of papers by Cheng [2] and Merkin [3,4], and it is therefore unnecessary to repeat the details here.

Our present objective is to predict the steady natural convection heat transfer from an impermeable vertical surface embedded in a saturated porous medium subject to a prescribed non-uniform wall temperature or to a prescribed non-uniform wall heat flux. We show that the governing equations possess a similarity solution only for a limited class of wall temperature distribution or heat flux rate distribution with a resulting ordinary differential equations. The procedure, as we apply it here, is close to that used by $\mathrm{Na}[5,6]$ who has developed a consistent theory based on a very efficient two-point finite-difference method, and the readers are referred to [5,6] for detailed analysis. Thus, the numerical solution of the boundary layer equations, which starts at $\xi=0$ ( $\xi$ measures distance along the wall) and proceeds along the plate up to $\xi=42.15$ to the required accuracy are obtained. The results are presented for both wall temperature distribution and wall heat flux distribution. We report the principal results in the form of tables of the heat transfer rates and of the excess surface temperature as a function of the parameter $\xi$. However, other quantities of interest that emerge from our calculations are the 
velocity and temperature fields but we do not present them here for the sake of brevity. Such solutions are not available in the existing literature and therefore they may serve as a reference against which other approximate solutions or emperimental data can be compared in the future. On the other hand, the present analysis reveals that this method can be applied to a wide range of non-similar problems in boundary-layer theory and other related areas.

\section{BASIC EQUATIONS}

We consider the natural convection about a heated semi-infinite vertical impermeable flat plate in an unbounded region of saturated porous medium with constant permeability $K$. We assume that the flow through the porous medium is governed by Darcy's law. It is also assumed that the Rayleigh number is large and that the Boussinesq approximation is applicable to the present problem. That is to say, variable fluid properties are negligible except in the buoyancy term which is directly responsible for the fluid motion. Then, the fundamental equations may be taken to be

$$
\begin{gathered}
\frac{\partial^{2} \psi}{\partial y^{2}}=\frac{g_{e} \beta K \rho_{x}}{\mu} \frac{\partial T}{\partial y} \\
\alpha \frac{\partial^{2} T}{\partial y^{2}}=\frac{\partial \psi \partial T}{\partial y} \frac{\partial T}{\partial x}-\frac{\partial \psi}{\partial x} \frac{\partial T}{\partial y}
\end{gathered}
$$

where $\psi$ is the stream function defined in the usual way, $x$ and $y$ are coordinates measuring distance along and normal to the plate respectively, $\alpha$ the equivalent thermal diffusivity, $\rho_{\infty}$ the density of the ambient fluid and $\mu$ the viscosity of convective fluid.

Equations (1) and (2) have to be solved subject to the boundary conditions

$$
\begin{aligned}
& y=0: \quad \frac{\partial \psi}{\partial x}=0, \quad T=T_{w}(x) \\
& y=\infty: \quad \frac{\partial \psi}{\partial y}=0, \quad T=T_{x}
\end{aligned}
$$

or

$$
\begin{gathered}
y=0: \quad \frac{\partial \psi}{\partial x}=0, \quad \frac{\partial T}{\partial y}=-\frac{q_{w}(x)}{k} \\
y=\infty, \quad \frac{\partial \psi}{\partial y}=0, \quad T=T_{x}
\end{gathered}
$$

where $T_{w}(x)$ designates temperature of the plate (variable), $T_{x}$ the temperature of the ambient fluid (constant), $q_{w}(x)$ the local heat transfer rate (variable) and $k$ thermal conductivity of the saturated porous medium.

In developing the method of solution, we shall refer to the above situations (3) and (4), respectively; but here we will restrict our attention to a rather specific form of $T_{w}(x)$ and $q_{w}(x)$.

\section{VARIABLE WALL TEMPERATURE $T_{w}(x)$}

The eqns (1)-(3) are firstly recast into non-dimensional form by introducing the variables

$$
\begin{gathered}
\psi=\alpha R_{a}^{1 / 2} \bar{\psi}, \quad \bar{y}=\frac{y}{L} R_{a}^{1 / 2}, \quad \bar{x}=\frac{x}{L} \\
\phi=\frac{T-T_{\infty}}{T_{w}(\bar{x})-T_{\infty}}, \quad S_{w}(\bar{x})=\frac{T_{w}(\bar{x})-T_{\infty}}{T_{r}-T_{\infty}} .
\end{gathered}
$$


The equations now read

$$
\begin{gathered}
\frac{\partial^{2} \psi}{\partial \bar{y}^{2}}=S_{w}(\bar{x}) \frac{\partial \theta}{\partial \bar{y}} \\
\frac{\partial^{2} \theta}{\partial \bar{y}^{2}}=\left\{\frac{\partial \theta}{\partial \bar{x}}+\theta \frac{\mathrm{d} \ln S_{w}(\bar{x})}{\mathrm{d} \bar{x}}\right\} \frac{\partial \bar{\psi}}{\partial \bar{y}}-\frac{\partial \theta}{\partial \bar{y}} \frac{\partial \bar{\psi}}{\partial \bar{x}}
\end{gathered}
$$

with boundary conditions

$$
\begin{aligned}
& \bar{y}=0: \quad \frac{\partial \bar{\psi}}{\partial \bar{x}}=0, \quad \theta=1 \\
& \bar{y}=\infty: \quad \frac{\partial \bar{\psi}}{\partial \bar{y}}=0, \quad \theta=0 .
\end{aligned}
$$

The further transformations which carry the inherent advantages are

$$
\begin{gathered}
\xi=\bar{x}, \quad \eta=\frac{\bar{y}}{\bar{x}^{1 / 2} S_{w}(\bar{x})^{-1 / 2}} \\
f(\xi, \eta)=\frac{\bar{\psi}}{\bar{x}^{1 / 2} S_{w}(\bar{x})^{1 / 2}}, \quad g(\xi, \eta)=\theta .
\end{gathered}
$$

The resulting equations are as follows

$$
\begin{gathered}
f^{\prime \prime}=g^{\prime} \\
g^{\prime \prime}+\frac{P(\xi)+1}{2} f g^{\prime}-P(\xi) f^{\prime} g=\xi\left(f^{\prime} \frac{\partial g}{\partial \xi}-g^{\prime} \frac{\partial f}{\partial \xi}\right)
\end{gathered}
$$

with the boundary conditions

$$
\begin{array}{cc}
\eta=0: & f(\xi, 0)=0, \quad g(\xi, 0)=1 \\
\eta=\infty: & f^{\prime}(\xi, \infty)=0, \quad g(\xi, \infty)=0 .
\end{array}
$$

Here the primes indicate differentiation with respect to $\eta$ and $P(\xi)$ is defined as

$$
P(\xi)=\frac{\xi}{S_{w}(\xi)} \frac{\mathrm{d} S_{w}(\xi)}{\mathrm{d} \xi}
$$

Integration of (10) subject to the boundary conditions (12b) results in

$$
f^{\prime}=g
$$

In view of (14), eqn (11) takes the final form

$$
f^{\prime \prime \prime}+\frac{P(\xi)+1}{2} f^{\prime \prime}-P(\xi)\left(f^{\prime}\right)^{2}=\xi\left(f^{\prime} \frac{\partial f^{\prime}}{\partial \xi}-f^{\prime \prime} \frac{\partial f}{\partial \xi}\right)
$$

with the boundary conditions

$$
\begin{gathered}
\eta=0: \quad f(\xi, 0)=0, \quad f^{\prime}(\xi, 0)=1 \\
\eta=\infty: \quad f^{\prime}(\xi, \infty)=0 .
\end{gathered}
$$


It becomes immediately evident, as is seen above, that in porous medium the velocity field which vanishes at infinity has been obtained under the same temperature distribution described by the eqn (14). The fluid is difficult to flow through the porous medium owing to the drag exerted by the medium and its rising motion due to buoyancy may be important to know for any geophysical problem.

The primary physical quantity of interest is the local Nusselt number $N u_{x}=h x / k$. With the aid of relations (5) and (9), and the use of Fourier's law $q_{w}=-k(\partial T / \partial y)_{y=0}$, the local Nusselt number can then be shown to be given by

$$
\frac{\mathrm{Nu}_{x}}{\mathrm{Ra}_{x}^{1 / 2}}=-\left(\frac{\partial^{2} f}{\partial \eta^{2}}\right)_{\eta=0}
$$

Hence, a complete solution of the problem involves solving eqn (15) with the respective boundary conditions (16). As it was mentioned in Section 1, we use a very efficient two-point finite-difference method to solve this equation. The solution procedure that we adopt for the eqns (15) and (16) can be found in detail in Refs. [5, 6]. For this reason, only a brief description of the numerical scheme is presented in Appendix.

Having derived appropriate equations, we proceed further with the analysis as follows.

\section{Case 1}

The first example is the special case in which $P(\xi)$ is constant, say $\lambda$. Consequently

$$
S_{w}(\xi)=A_{1} \xi^{\lambda} \text { and hence } T_{w}(x)=T_{\infty}+A x^{\lambda}
$$

where $A_{1}$ and $A$ are constants. The problem becomes similar and the r.h.s. of (15) becomes zero since for this case $f$ is independent of $\xi$; eqn (15) reduces to that of Cheng and Minkowycz[1]

$$
f^{\prime \prime \prime}+\frac{\lambda+1}{2} f f^{\prime \prime}-\lambda\left(f^{\prime}\right)^{2}=0
$$

with the boundary conditions

$$
f(0)=0, \quad f^{\prime}(0)=1, \quad f^{\prime}(\infty)=0 .
$$

\section{Case 2}

Another specific form at our disposal of $S_{w}(\bar{x})$ is

$$
S_{w}(\bar{x})=1+\bar{x}^{n}
$$

whereupon (13) reduces to

$$
P(\xi)=\frac{\xi}{S_{w}(\xi)} \frac{\mathrm{d} S_{w}(\xi)}{\mathrm{d} \xi}=\frac{n \xi^{n}}{1+\xi^{n}}
$$

A summary of the numerical results for the heat transfer rate when $n=1$ and 2 are given in Table 1.

\section{Case 3}

If the wall temperature varies exponentially with the distance along the wall then in terms of the variables defined in (5), one has

$$
S_{w}(\bar{x})=\mathrm{e}^{n \bar{x}}
$$

from which

$$
P(\xi)=\frac{\xi}{S_{w}(\xi)} \frac{\mathrm{d} S_{w}(\xi)}{\mathrm{d} \xi}=n \xi
$$


Table 1. Tabulation of $N u_{x} / R a_{x}^{1 / 2}$ for various $\xi$ s in Case 2

\begin{tabular}{|ccc|}
\hline$\xi$ & $n=1$ & $n=2$ \\
\hline 0.00 & 0.4438 & 0.4438 \\
0.11 & 0.5035 & 0.4551 \\
0.29 & 0.5750 & 0.5164 \\
0.56 & 0.6535 & 0.6734 \\
0.99 & 0.7315 & 0.9139 \\
1.66 & 0.8018 & 1.1292 \\
2.71 & 0.8597 & 1.2540 \\
4.35 & 0.9039 & 1.3101 \\
6.91 & 0.9357 & 1.3330 \\
10.90 & 0.9577 & 1.3422 \\
17.15 & 0.9724 & 1.3460 \\
26.91 & 0.9821 & 1.2475 \\
42.15 & 0.9885 & 1.3481 \\
\hline
\end{tabular}

Table 2. Tabulation of $N u_{x} / R u_{x}^{1 / 2}$ for various $\xi$ s in Case 3

\begin{tabular}{|cccc|}
\hline$\xi$ & $\mathrm{n}=0.1$ & $\mathrm{n}=0.5$ & $\mathrm{n}=1$ \\
\hline 0.00 & 0.4438 & 0.4438 & 0.4438 \\
0.11 & 0.4504 & 0.4766 & 0.5086 \\
0.29 & 0.4607 & 0.5262 & 0.6039 \\
0.56 & 0.4766 & 0.6002 & 0.7400 \\
0.99 & 0.5011 & 0.7080 & 0.9279 \\
1.66 & 0.5384 & 0.8604 & 1.2772 \\
2.71 & 0.5946 & 1.0683 & 1.4959 \\
4.35 & 0.6780 & 1.3410 & 1.8936 \\
6.91 & 0.7984 & 1.6874 & 2.3864 \\
10.90 & 0.9670 & 2.1201 & 2.9984 \\
17.15 & 1.1944 & 2.6589 & 3.7603 \\
26.91 & 1.4900 & 3.3306 & 4.7102 \\
42.15 & 1.8642 & 4.1688 & 5.8955 \\
\hline
\end{tabular}

Numerical data for $n=0.1,0.5$ and 1 are shown in Table 2 .

4. VARIABLE HEAT FLUX RATE $q_{w}(x)$

We now define the following dimensionless variables

$$
\begin{gathered}
\psi=\alpha R a^{* 1 / 3} \bar{\psi}, \quad \vec{x}=\frac{x}{L}, \quad \bar{y}=\frac{y}{L} R a^{* 1 / 3} \\
\theta=\frac{T-T_{x}}{\theta_{r}(\bar{x})}, \quad \theta_{r}(\bar{x})=\frac{q_{w}(\bar{x}) L}{k R a^{* 1 / 3}} .
\end{gathered}
$$

When (25) is introduced into (1), (2) and (4) it leads to

$$
\begin{gathered}
\frac{\partial^{2} \bar{\psi}}{\partial \bar{y}^{2}}=\frac{\partial \theta}{\partial \bar{y}} \\
\frac{\partial^{2} \theta}{\partial \bar{y}^{2}}=\frac{\partial \bar{\psi}}{\partial \bar{y}} \frac{\partial \theta}{\partial \bar{x}}-\frac{\partial \bar{\psi}}{\partial \bar{x}} \frac{\partial \theta}{\partial \bar{y}}+\frac{\mathrm{d} \ln \theta_{r}(\bar{x})}{\mathrm{d} \bar{x}}\left(\theta \frac{\partial \bar{\psi}}{\partial \bar{y}}-\frac{1}{2} \bar{\psi} \frac{\partial \theta}{\partial \bar{y}}\right)
\end{gathered}
$$

with the boundary conditions

$$
y=0: \quad \frac{\partial \bar{\psi}}{\partial \bar{x}}=0, \quad \frac{\partial \theta}{\partial \bar{y}}=-1
$$




$$
y=\infty: \quad \frac{\partial \bar{\psi}}{\partial \bar{y}}=0 .
$$

By introducing an appropriate transformations

$$
\xi=\bar{x}, \quad \eta=\frac{\bar{y}}{\bar{x}^{1 / 3}}, \quad f(\xi, \eta)=\frac{\bar{\psi}}{\bar{x}^{2 / 3}}, \quad g(\xi, \eta)=\frac{\theta}{\bar{x}^{1 / 3}}
$$

we obtain instead of (26) and (27) the equation

$$
f^{\prime \prime \prime}+\left\{\frac{2}{3}+\frac{Q(\xi)}{2}\right\} f f^{\prime \prime}-\left\{\frac{1}{3}+Q(\xi)\right\}\left(f^{\prime}\right)^{2}=\xi\left(f^{\prime} \frac{\partial f^{\prime}}{\partial \xi}-f^{\prime \prime} \frac{\partial f}{\partial \xi}\right)
$$

with the boundary conditions

$$
\begin{gathered}
\eta=0: \quad f(\xi, 0)=0, \quad f^{\prime \prime}(\xi, 0)=-1 \\
\eta=\infty: \quad f^{\prime}(\xi, \infty)=0 .
\end{gathered}
$$

Here we have denoted

$$
Q(\xi)=\frac{\xi}{\theta_{r}(\xi)} \frac{\mathrm{d} \theta_{r}(\xi)}{\mathrm{d} \xi}
$$

In terms of the transformed variables (25) and (29), we can define a non-dimensional excess surface temperature $T_{s}$ by

$$
T_{s}=\theta(\xi, 0)=\left(\frac{\partial f}{\partial \eta}\right)_{\eta=01} .
$$

\subsection{Similar flow}

(i) If $q_{w}=$ constant, then $Q(\xi)=0$ and (30) is reduced to an ordinary differential equation, namely

$$
f^{\prime \prime \prime}+\frac{2}{3} f f^{\prime \prime}-\frac{1}{3}\left(f^{\prime}\right)^{2}=0
$$

with the boundary conditions

$$
f(0)=0, \quad f^{\prime \prime}(0)=-1, \quad f^{\prime}(\infty)=0 .
$$

Thus, the solution is similar. It should be mentioned that eqns (34) and (35) become identical with those of Merkin [4] (eqns (20) and (21) in his paper) by a proper choice of $f$ and $\eta$.

Refering to definition of $\theta_{r}(x)$ from (25b), it follows

$$
\theta_{r}(\bar{x})=\frac{q_{w}(\bar{x}) L}{k R a^{* / 3}}=\frac{q_{w} \bar{x} L}{k\left\{\frac{g_{e} \beta \rho_{x} q_{w}(\bar{x}) K L^{2}}{\alpha \mu k}\right\}^{1 / 3}}=C\left\{q_{w}(\bar{x})\right\}^{2 / 3}
$$

and (32) could be written as

$$
Q(\xi)=\frac{\xi}{\theta_{r}(\xi)} \frac{\mathrm{d} \theta_{r}(\xi)}{\mathrm{d} \xi}=\frac{2}{3} \frac{\xi}{q_{w}(\xi)} \frac{\mathrm{d} q_{w}(\xi)}{\mathrm{d} \xi}
$$

(ii) When $q_{w}(\xi)$ is proportional to $\xi^{m}$, i.e.

$$
q_{w}(\xi)=C_{1} \xi^{m}
$$


(37) yields

$$
Q(\xi)=\frac{2}{3} m
$$

where $C_{1}$ and $m$ are constants. The problem is again similar and equation (30) reduces to

$$
f^{\prime \prime \prime}+\left(\frac{2}{3}+\frac{m}{2}\right) f^{\prime \prime}-\left(\frac{1}{3}+\frac{2}{3} m\right)\left(f^{\prime}\right)^{2}=0
$$

subject to the same boundary conditions (35).

It may be noted that these are only the cases where similar solutions are possible.

Some numerical values of $T_{s}$ for different $m$ 's are presented in Table 3. We remark here that our result for the case of constant heat flux $(m=0)$ is in very good agreement with that from Ref. [4] if one multiplies the latter by the factor 2 .

\subsection{Non-similar flow}

To generate the solution of (30) subject to (31) in the general case we use the same method as in the specified $T_{w}(x)$ case (see Appendix). But, now the first approximation $(\nu=1)$ of (A15) for all examples are different of course and are given by

$$
f_{j}^{(1)}=\frac{1}{2} \tau-\frac{1}{2} \tau^{2}+\frac{1}{6} \tau^{3}, \quad u_{j}^{(1)}=\frac{1}{2}-\tau+\frac{1}{2} \tau^{2} \quad v_{j}^{(1)}=\tau-1
$$

\section{Case 4}

Consider next that $q_{w}(\bar{x})$ has the specific form

$$
q_{w}(\bar{x})=1+\bar{x}^{n} .
$$

We then see from (36) that

$$
\theta_{r}(\bar{x})=C\left(1+\bar{x}^{n}\right)^{2 / 3}
$$

Table 3. Tabulation of $T$, for various $m$ 's

\begin{tabular}{|ll|}
\hline & $T_{0}$ \\
\hline 0 & 1.2955 \\
1 & 0.9223 \\
2 & 0.7817 \\
\hline
\end{tabular}

Table 4. Tabulation of $T_{3}$ for various $\xi^{\prime} s$ in Case 4

\begin{tabular}{|ccc|}
\hline$\xi$ & $\mathrm{n}=1$ & $\mathrm{n}=2$ \\
\hline 0.00 & 1.2955 & 1.2955 \\
0.11 & 1.2676 & 1.2910 \\
0.29 & 1.2334 & 1.2664 \\
0.56 & 1.1946 & 1.2017 \\
0.99 & 1.1547 & 1.0958 \\
1.66 & 1.1172 & 0.9911 \\
2.71 & 1.0850 & 0.9235 \\
4.35 & 1.0595 & 0.8898 \\
6.91 & 1.0405 & 0.8751 \\
10.90 & 1.0270 & 0.8691 \\
17.15 & 1.0177 & 0.8666 \\
26.91 & 1.0115 & 0.8656 \\
42.15 & 1.0074 & 0.8652 \\
\hline
\end{tabular}


Table 5. Tabulation of $T$, for various $\xi$ 's in Case 5

\begin{tabular}{|rrr|}
\hline $\boldsymbol{\xi}$ & $\mathrm{n}=0.5$ & $\mathrm{n}=1$ \\
\hline 0.00 & 1.2955 & 1.2955 \\
0.11 & 1.2804 & 1.2656 \\
0.29 & 1.2575 & 1.2219 \\
0.56 & 1.2236 & 1.1603 \\
0.99 & 1.1747 & 1.0777 \\
1.66 & 1.1070 & 0.9752 \\
2.71 & 1.0189 & 0.8599 \\
4.35 & 0.9136 & 0.7442 \\
6.91 & 0.8003 & 0.6393 \\
10.90 & 0.6915 & 0.5492 \\
17.15 & 0.5950 & 0.4722 \\
26.91 & 0.5120 & 0.4064 \\
42.15 & 0.4409 & 0.3499 \\
\hline
\end{tabular}

and consequently, using (37) we have

$$
Q(\xi)=\frac{\xi}{\theta_{r}(\xi)} \frac{\mathrm{d} \theta_{r}(\xi)}{\mathrm{d} \xi}-\frac{2}{3} \frac{n \xi^{n}}{1+\xi^{n}}
$$

Values of $T_{s}$ for $n=1$ and 2 are given in Table 4

\section{Case 5}

Finally, we assume that heat flux rate from the wall varies exponentially with $x$, i.e.

$$
q_{w}(\bar{x})=e^{n \bar{x}} .
$$

Hence

$$
\theta_{r}(\bar{x})=C \mathrm{e}^{2 / 3 n \bar{x}} \text { so that } Q(\xi)=\frac{2 n}{3} \xi \text {. }
$$

As in the previous examples, Table 5 is included where the solution of the problem for $n=0.5$ and 1 are tabulated.

\section{CONCLUSION}

Some natural convection problems for a semi-infinite vertical flat plate embedded in a highly saturated porous medium were solved by a very efficient and convenient numerical method. Since there apparently no results in the existing literature with which to compare our own findings we have summarized the numerical data in Tables for both the heat transfer rate and excess surface temperature. This is essentially accurate new information against to test the approximate methods.

\section{NOMENCLATURE}

$C$ constant $=\left(\alpha \mu L / g_{e} k^{2} \beta \rho, K\right)^{1 / 3}$

$g_{e}$ acceleration due to gravity

$h$ local heat transfer coefficient $=q_{w} /\left(T_{w^{\prime}}-T_{x}\right)$

$k$ thermal conductivity of the saturated porous medium

$K$ permeability of the porous medium

$L$ length of the plate

$m, n$ constants

$q_{\mathrm{w}}$ local heat transfer rate

$R a, R a^{*}$ modified Rayleigh numbers, $R a=g_{e} \beta \rho_{x}\left(T_{r}-T_{x}\right) K L / \alpha \mu$ and $R a^{*}=g_{e} \beta \rho_{x} q_{w^{*}}(x) K L^{2} / \alpha \mu k$

$R a_{x} \quad$ modified local Rayleigh number $=g e \beta \rho_{x}\left(T_{w^{*}}-T_{x}\right) K x / \alpha \mu$

$T$ temperature

$T_{s} \quad$ excess surface temperature (non-dimensional) $=\left\{g_{\mathrm{e}} \beta \rho_{x} K q_{u}(\xi) L^{2} / \alpha \mu k\right\}^{1 / 7}\left(T_{u}-T_{*}\right) /\left\{q_{w}(\xi) L / k\right\} \xi^{1 / 3}$

$x$ vertical coordinate 
$y$ horizontal coordinate

$\alpha$ echivalent thermal diffusivity

$\beta$ coefficient of thermal expansion

$\mu$ viscosity of convective fluid

$\rho_{x}$ density of convective fluid

$\psi$ stream function

\section{Subscripts}

$r$ reference

$w$ wall condition

$\infty$ ambient condition

\section{REFERENCES}

[1] P. CHENG and W. J. MINKOWYCZ, J. Geophys. Res. 82, 2040 (1977).

[2] P. CHENG, Int. J. Heat Mass Transfer 20, 201 (1977).

[3] J. H. MERKIN, Int. J. Heat Mass Transfer 21, 1499 (1978).

[4] J. H. MERKIN, J. Engng. Math. 14, 301 (1980).

[5] T. Y. NA, Appl. Sci. Res. 33, 519 (1978).

[6] T. Y. NA, Computational Methods in Engineering Boundary Value Problems. Academic Press, New York (1979).

(Received 15 December 1981)

\section{APPENDIX}

One of the basic ideas of the two-point finite-difference method is to replace the governing eqn (15) by a first-order system

$$
\begin{gathered}
f^{\prime}=u \\
u^{\prime}=v \\
v^{\prime}+\frac{P(\xi)+1}{2} f v-P(\xi) u^{2}=\xi\left(u \frac{\partial u}{\partial \xi}-v \frac{\partial f}{\partial \xi}\right)
\end{gathered}
$$

with the boundary conditions

$$
\eta=0: \quad f(\xi, 0)=0, \quad u(\xi, 0)=1 \quad \eta=\infty: \quad u(\xi, \infty)=0 .
$$

To discretize the equations we set up a rectangular net with the net points characterized by

$$
\left.\begin{array}{c}
\xi_{0}=0 \quad \xi_{n}=\xi_{n-1}+k_{n} \quad(n=1,2, \ldots, N) \\
\eta_{0}=0, \quad \eta_{J}=\eta_{j-1}+h_{j} \quad(j=1,2, \ldots, J), \quad \eta_{J}=\eta_{\infty}
\end{array}\right\}
$$

where $h_{j}$ and $k_{n}$ are step sizes.

Let us consider can (A3) first and replace the derivatives with respect to $\xi$ by the finite-difference. After some algebra one then obtains

$$
l^{n}-\alpha_{n}\left\{\left(u^{n}\right)^{2}-v^{n} f^{n}-v^{n-1} f^{n}+f^{n-1} v^{n}\right\}=R^{n-1}
$$

where

$$
\begin{gathered}
L=\text { l.h.s. of } \\
\alpha_{n}=\xi_{n-1 / 2} / k_{n} \\
R^{n-1}=\alpha_{n}\left\{v^{n-1} f^{n-1}-\left(u^{n-1}\right)^{2}\right\}-L^{n-1} .
\end{gathered}
$$

Replacing $L$ 's by their complete form, and dropping the superscript " $n$ ", eqns (A1)-(A3) become

$$
\begin{gathered}
f^{\prime}=u \\
u^{\prime}=v \\
v^{\prime}+\frac{P(\xi)+1}{2} f v-P(\xi) u^{2}-\alpha_{n}\left(u^{2}-f v-v^{n-1} f+f^{n-1} v\right)=R^{n-1}
\end{gathered}
$$

where

$$
R^{n-1}=\alpha_{n}\left\{v^{n-1} f^{n-1}-\left(u^{n-1}\right)^{2}\right\}-\left\{\left(v^{n-1}\right)^{\prime}+\frac{P(\xi)+1}{2} f^{n-1} v^{n-1}-P(\xi)\left(u^{n-1}\right)^{2}\right\}
$$

If we now replace $\eta$-derivatives by the finite-difference it follows that

$$
f_{j}-f_{i-1}-\frac{h_{j}}{2}\left(u_{j}+u_{j-1}\right)=0
$$




$$
\begin{gathered}
u_{1}-u_{1-1}-\frac{h_{j}}{2}\left(v_{j}+v_{j-1}\right)=0 \\
v_{j}-v_{j-1}+\frac{P(\xi)+1}{2} h_{1} f_{j-1 / 2} v_{j-1 / 2}-P(\xi) h_{j}\left(u_{j-1 / 2}\right)^{2}-\alpha_{n} h_{1}\left\{\left(u_{j-1 / 2}\right)^{2}-v_{j-1 / 2} f_{j-1 / 2}-v_{j-1 / 2}^{n-1}+f_{j-1 / 2}^{n-1} v_{j-1 / 2}\right\}=S_{j-1 / 2}^{n-1}
\end{gathered}
$$

with the boundary conditions

$$
u_{1}=0, \quad f_{1}=1, \quad u_{j}=0
$$

where

$$
S_{j-1 / 2}^{n-1}=\alpha_{n} h_{j}\left\{v_{j-1 / 2}^{n-1} f_{j-1 / 2}^{n-1}-\left(u_{j-1 / 2}^{n-1}\right)^{2}\right\}-\left\{\left(v_{j}^{n-1}-v_{j-1}^{n-1}\right)+\frac{P(\xi)+1}{2} h_{j} j_{1-1 / 2}^{n-1} v_{j-1 / 2}^{n-1}-P(\xi) h_{j}\left(u_{j-1 / 2}^{n-1}\right)^{2}\right\} .
$$

Further we put

$$
f_{i}^{(\nu+1)}=f_{i}^{(v)}+\delta f_{i}^{(\nu)}, \text { etc. }
$$

but, for convenience, we drop two superscripts. So, let us replace (A14) by

$$
f_{j}=f_{i}^{(\nu)}+\delta f_{j}, \text { etc. }
$$

and introduce (A15) and (A10), (A11) and (A12); we get

$$
\begin{gathered}
\delta f_{j}-\delta f_{j-1}-\frac{h_{j}}{2}\left(\delta u_{j}+\delta u_{j-1}\right)=\left(a_{1}\right)_{j} \\
\delta u_{j}-\delta u_{j-1}-\frac{h_{j}}{2}\left(\delta v_{j}+\delta v_{j-1}\right)=\left(a_{2}\right)_{j} \\
\left(b_{1}\right)_{j} \delta f_{j-1}+\left(b_{2}\right)_{j} \delta f_{j}+\left(b_{3}\right)_{j} \delta u_{j-1}+\left(b_{4}\right)_{j} \delta u_{j}+\left(b_{5}\right)_{j} \delta v_{j-1}+\left(b_{6}\right)_{j} \delta v_{j}=\left(a_{3}\right)_{j}
\end{gathered}
$$

with the boundary conditions

$$
\delta f_{1}=0, \quad \delta v_{1}=0, \quad \delta u_{j}=0 .
$$

In the above equations we have denoted

$$
\begin{aligned}
& \left(a_{1}\right)_{j}=f_{j-1}-f_{j}+\frac{h_{j}}{2}\left(u_{1}+u_{j-1}\right) \\
& \left(a_{2}\right)_{j}=u_{j-1}-u_{j}+\frac{h_{I}}{2}\left(v_{j}+v_{l-1}\right)
\end{aligned}
$$

$\left(a_{3}\right)_{j}=S_{j-1 / 2}^{n-1}-v_{j}+v_{j-1}-\frac{P(\xi)+1}{2} h_{j} f_{j-1 / 2} v_{1-1 / 2}+P(\xi)\left(u_{j-1 / 2}\right)^{2} h_{j}+\alpha_{n} h_{1} \quad\left\{\left(u_{1} 1 / 2\right)^{2}-v_{1} 1 / 2 f_{1} 1 / 2-v_{j}^{n-1 / 2} f_{1} 1 / 2+f_{j-1 / 2}^{n-1} v_{1-1 / 2}\right\}$

$$
\begin{gathered}
\left(b_{1}\right)_{j}=\left(b_{2}\right)_{j}=\frac{P(\xi)+1}{4} h_{1} v_{1-1 / 2}+\frac{1}{2} \alpha_{n} h_{j}\left(v_{1-1 / 2}+v_{j-1 / 2}^{n-1}\right. \\
\left(b_{3}\right)_{j}=\left(b_{4}\right)_{j}=\left\{P(\xi)+\alpha_{n}\right\} h_{j} u_{j-1 / 2} \\
\left(b_{5}\right)_{1}=-1+\frac{P(\xi)+1}{4} h_{j} f_{j-1 / 2}-\frac{1}{2} \alpha_{n} h_{j}\left(f_{1-1 / 2}^{n-1}-f_{1-1 / 2}\right) \\
\left(b_{6}\right)_{1}=\left(b_{5}\right)_{j}+2 .
\end{gathered}
$$

For the first approximation $(\nu=1)$ of $(\mathrm{A} 15)$ we use

$$
\left.\begin{array}{c}
f_{1}^{(1)}=\tau-\tau^{2}+\frac{\tau^{3}}{3}, u_{j}^{(1)}=1-2 \tau+\tau^{2} \\
v_{j}^{(1)}=2(\tau-1)
\end{array}\right\}
$$

in which $\tau=\eta / \eta_{\max }$. Then, eqns (A16)-(A18), subject to (A19) are solved by the method of factorization (see[6], Chap. 6 , pp. 126-132) to get $\delta f_{j}, \delta u_{j}$ and $\delta v_{j}$ for all j's. The next approximation can be obtained as

$$
f_{j}^{(2)}=f_{j}^{(1)}+\delta f_{j}, \text { etc. }
$$

and this will be continued until the solution converges with desired accuracy. 